\title{
Multibody model of fruit harvesting trucks: comparison with experimental data and rollover analysis
}

\author{
Stefano Melzi, ${ }^{1}$ Edoardo Sabbioni, ${ }^{1}$ Michele Vignati, ${ }^{1}$ Maurizio Cutini, ${ }^{2}$ Massimo Brambilla, ${ }^{2}$ \\ Carlo Bisaglia, ${ }^{2}$ Eugenio Cavallo ${ }^{3}$
}

${ }^{1}$ Department of Mechanical Engineering, Politecnico di Milano; ${ }^{2}$ Consiglio per la ricerca in agricoltura e l'analisi dell'economia agraria (CREA), Treviglio (BG); ${ }^{3}$ Institute for Agricultural and Earthmoving Machines (IMAMOTER), National Research Council of Italy (CNR), Torino, Italy

\begin{abstract}
Fruit harvesting trucks are used to easy and speed-up the work of agricultural operators. These vehicles are provided with a moving cargo bed, which can be raised up to 3 meters from the ground so that workers are closer to the plants top. Due to factors like height of centre of gravity and operation on soft and irregular soil, these vehicles present several safety issues. This research, carried out inside a project funded by INAIL (Italian National Institute for Insurance against Accidents at Work), analysed the stability of fruit harvesting trucks with particular focus on rollover risk. Experimental tests were carried out to characterise the response of these vehicles. Multibody models of two trucks were then developed and used to determine the rollover angle along a generic direction considering the effect of vehicle configuration and of tire-soil stiffness.
\end{abstract}

\section{Introduction}

Rough-terrain work platforms for orchard's operations are self-propelled machine designed to work on unimproved natural

Correspondence: Michele Vignati, Department of Mechanical Engineering, Politecnico di Milano, via La Masa 1, 20156 Milano, Italy. E-mail: michele.vignati@polimi.it

Key words: Multibody modelling; harvesting truck; rollover; mass distribution; tire-soil stiffness.

Acknowledgements: the study was carried out within the PROMOSIC project, within the framework of the BRIC 2015 call funded by INAIL (Italian National Institute for Insurance against Accidents at Work). The authors are grateful to Mr. G. Rozzoni, I. Carminati and A. Filisetti for the operative support during the tests campaign.

Received for publication: 12 July 2017.

Accepted for publication: 29 November 2017.

CC Copyright S. Melzi et al., 2018

Licensee PAGEPress, Italy

Journal of Agricultural Engineering 2018; XLIX:755

doi:10.4081/jae.2018.755

This article is distributed under the terms of the Creative Commons Attribution Noncommercial License (by-nc 4.0) which permits any noncommercial use, distribution, and reproduction in any medium, provided the original author(s) and source are credited. terrain or disturbed terrain (EN 16952, 2016). They generally consist of a chassis with a variable height work platform with lateral extending structures. They are usually equipped with hydrostatic transmission acting on a 2 axles and 4 wheels drive propulsion system. A Diesel engine, or more recently an electrical engine, powers the propulsion system and the platform elevation mechanisms. Driving and elevation controls are placed on the platform and traveling is allowed forward and rearward with the platform at any of its height. The machine moves very slowly (less than 2 $\mathrm{km} / \mathrm{h}$ ) from a working station to the next and, in some circumstances, the operational speed is so low that the driver is used to leave the driving controls to take part in the picking or pruning process. The speed of the machines in transfer mode is usually not higher than $15 \mathrm{~km} / \mathrm{h}$. Among this type of vehicles, fruit harvesting trucks (FHTs) are widely employed to improve productivity in agricultural operations. Basically it is a two-axle vehicle with a platform (cargo bed), which can be raised from the ground level so that the operators can easily reach the upper part of the plants. The forward speed and the direction of the vehicle can be controlled directly from the cargo bed: this allows moving the operators to the next plant when the harvest on the previous is completed. The moving cargo bed is also provided with two telescopic platforms, which extend laterally; this particular feature allows the operators to get closer to the plants even when the distance between rows is about to 4 meters.

FHTs generally operate on flat ground moving along the interrows of the orchard; anyway it is possible to use them also when moderate slopes are present especially when the vehicles are equipped with a self-levelling system allowing compensating for the ground gradient keeping the cargo bed in horizontal position.

These machines are intended to move at least two persons to make easier for them to carry out fruit picking, pruning and thinning or other operations, such as anti-hail net laying, because closer to the working area. Depending on the operations the machine is intended for, it can be equipped with additional equipment such as elevators and rails to load, carry and unload fruit containers (bins), independent levels for front/rear or left/right portion of the platform, picking assistance system, pneumatic or hydraulic pruning system. Since their introduction the picking platform such as the FHT and the mobile elevating work platforms have contributed to make easier and less strenuous such operations and to improve the labour productivity in the fruit farming (Coppock and Jutras, 1960). Thus, the adoption of picking platforms has reduced energy and strength demands opening up the opportunities for a larger percentage of male and much more female workers, especially, for the harvesting operations. Furthermore, implementation of picking platform in Europe and the US has reduced the exposure to fall hazard from ladder climbing and descending and to musculoskeletal disorders from excessive carrying and 
lifting load from bags or baskets (Fathallah, 2010).

Nevertheless, the FHTs present several factors of risks for the operators. In relation to FHT, the Mutualitè Sociale Agricole, the second largest social cover system in France, recorded 325 accidents from 2002 to 2009 and 2 deaths between 1995 and 2009, one of them because of loss of stability of the machine when working on a road shoulder.

The rollover stability of FHT is highly affected by the position of additional masses, such as operators and fruit containers, and the height that the platform can reach, up to 3 meters above the ground level. When one of the lateral extending structure of the platform is open and all the operators work on that side, the centre of gravity moves laterally. In such condition, the lateral position of the vehicle overall centre of gravity (c.o.g.) when the vehicle is driven on ground where holes, slopes and soil deformation may jeopardise stability even at low speed. Furthermore, when a maximum admissible inclination angle is recommended by the manufacturer of the machine, the lack, or fault, of an appropriate device can increase the risk of loss of stability because of the inaccuracy of the operators in estimating tilting angles (Görücü et al., 2014; Cavallo et al., 2015). Although rollover stability of agricultural vehicles is widely investigated in the technical literature (Liu and Ayers, 1999; Gravalos et al., 2011; Jung et al., 2013; Franceschetti et al., 2014; Vidoni et al., 2015; Wang et al., 2016), and several indexes were proposed, at best of authors' knowledge, no specific studies were carried out concerned with FHTs, which are the focus of the present paper.

The study aims at developing a procedure for assessing the safety requirements of FHTs and, in particular, to the definition of a standard procedure to determine the rollover angle. A numericalexperimental approach was used to investigate rollover of FHTs. Considering the experimental side, field tests were performed to analyse the typical operating conditions of these vehicles: forward speed, maximum roll angle, distribution of loads on the cargo bed. In addition, two FHTs were tested on a tilting table for identifying the maximum angle allowed before rollover to assess effect of the front axle suspension on rollover stability. Other indoor tests were performed to determine inertial and elastic parameters of the trucks. Based on the experimental data, a multibody (MB) model of the two vehicles was developed in Simulink/SimMechanics environment. The models reproduce the main features of the FHT, i.e.: the moving cargo bed, the telescopic platforms, and the variable position of the operators and of the loads. Model parameters like masses, compliance in joints and tire deformation, were tuned on the basis of the tests performed on the tilting table. A virtual test bench was then set-up allowing changing the inclination of the ground along a generic axis (i.e. not just longitudinal or lateral) so that the maximum angle allowed before rollover along a generic direction could be identified. A sensitivity analysis was eventually carried out to assess the effect of suspension system of the front pivoting axle and of tire stiffness on FHTs rollover limit. Rollover calculated by the MB model was compared with the value predicted by standards ISO 16231-1 and ISO 16231-2 to assess the influence of suspension, tire, and joint compliance. For what emerges from simulation, the tyre stiffness can considerably affect the rollover risk. In particular, for low inflated tyres, the rollover critical angle can be considerably smaller than the one computed according to standard.

\section{Experimental tests}

\section{Field tests}

Sessions of field tests were performed to analyse the typical operating conditions of FHTs. Tests were carried out in two fields considering different track models. The terrain of both the fields was almost flat with an average slope of $1^{\circ}$, suitable for gravity irrigation. The distance between rows was equal to 4 meters: the operations were thus carried out with the lateral platforms extended up to 3.50 meters. Under normal operating conditions, the forward speed of the vehicles was in the range $0.1-2 \mathrm{~km} / \mathrm{h}$; the vehicle roll angles, measured putting the sensor on the chassis, presented a maximum recorded value during the operations of about $2.7^{\circ}$.

The workers operated close to the edge of the platforms: their feet were in fact almost always in contact with the toe barrier. They used boxes fastened to the balustrade to collect fruits. When full (mass is about $8 \mathrm{~kg}$ ) these boxes were emptied in a big bin positioned at the centre of the cargo bed. The bin has standard dimensions $(1200 \times 1100 \times 630 \mathrm{~mm})$ with a maximum mass of $400 \mathrm{~kg}$. According to the results of the field test, dynamic effects due to speed or accelerations are likely negligible. Observation of the operation during field test makes evident the typical position and the weight of the operators and the payload. This data are summarised in Figure 1 and were used to set the scenarios for tilting
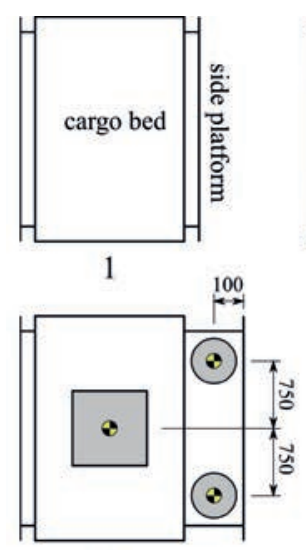

3

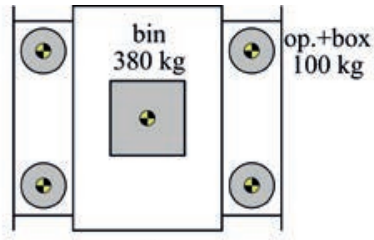

2

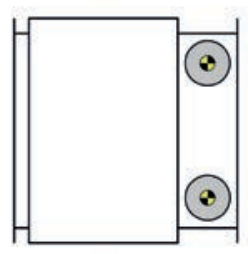

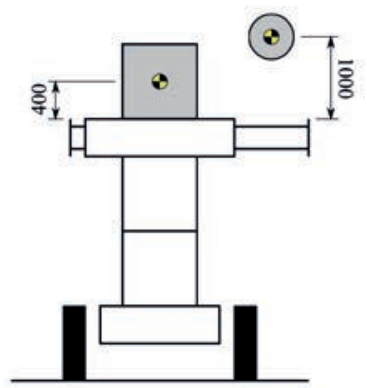

Figure 1. Payload and working conditions. Position and mas of fruit bin and operators+box. 
tests and simulations. Specifically, the following conclusions could be drawn: i) operators are placed always near the barriers; ii) operators mass must be increased of the mass of buckets attached to the barriers (operators mass and c.o.g. height from cargo bed is taken in accordance to ISO 3411, UNI 1459 and ISO 22915-1/2); iii) the mass of the bin must be possibly taken into account, placed at the centre of the cargo bed.

\section{Tilting tests}

Laboratory tests were carried out in order to define the tilt angle of the platform at which rollover occurs for the two FHTs considered in the most critical working conditions. The experimental setup, in accordance with standard ISO 16231-1 and ISO 16231-2, consists in positioning the FHT on a tilting platform with different orientations and measuring the inclination angle of the platform at which the rollover occurs.

Figure 2 shows examples of the performed tilting tests. The following prescriptions were adopted when conducting the experimental tilting tests: i) the platform tilting angle was increased slowly in order to reduce as much as possible any dynamic effect on rollover; ii) deformation of the platform was continuously monitored and no deformation has been registered during the test; iii) the fluid tanks (fuel and hydraulic oil) were in running order conditions thus filled properly; iv) tires were inflated according to the manufacturer's recommended pressure; v) downstream wheels were laterally blocked in order to limit lateral sliding during the test. The height of blocking devices was less than $10 \%$ of tire nominal radius as prescribed by ISO 16231-1; vi) chains were used to prevent full rollover after wheel detachment from ground, the bin was fixed to be in the centre of the platform for safety issues; vii) tests have been carried tilting the vehicle in the direction of the most critical side (i.e. the more loaded side).

Based on the experimental field tests described in the Field tests section, the following conditions were considered worthy of specific attention from rollover point of view and thus investigated on the tilting table, see Figure 1: i) condition 1: cargo bed at the maximum allowed height, side platforms closed, no additional loads; ii) condition 2: cargo bed at the maximum allowed height, side extension completely opened, 4 workers ( $100 \mathrm{~kg}$ each), two on each side of the truck, and one fruit bin, fully loaded, at the centre of the cargo bed $(380 \mathrm{~kg})$; iii) condition 3: cargo bed at the maximum allowed height, right side platforms opened, left closed, 2 workers (100 kg each) on right side of the truck and fruit bin, fully loaded, at the centre of the cargo bed $(380 \mathrm{~kg})$; iv) condition 4 : cargo bed at the maximum allowed height, right side platforms opened, left closed, 2 workers (100 kg each) on right side of the truck and no fruit bin.

All the tests were performed tilting the table towards the right (i.e. load at downstream).

Condition 1 defines the reference condition for rollover since it represents the less critical condition when the platform is placed at the maximum height.

Conditions 2, 3 and 4 investigate the influence of load distribution on rollover limit. Specifically, conditions 3 and 4 analyse the effect of an asymmetric load with and without the fruit bin in the centre of the cargo bed, while conditions 2 investigates the effect of a symmetric increase of the mass over the cargo bed (bin plus operators).

A longitudinal rollover test was also performed: the vehicle was placed with rear axle downstream and the tilt table was rotated until detachment of front axle from the ground. Only condition 1 was considered in the longitudinal rollover test.

\section{Tested vehicles}

Field and laboratory tests were performed on two FHTs. One FHT was equipped with a front pivoting axle with springs limiting the swivelling of the suspension. The other, instead, had a swivelling front suspension without any limiting device, see Figure 3. This means that the front axle is free to rotate with respect to the vehicle chassis for a wide-angle range (i.e. until axle/tires get in touch with the chassis). The rear axle of both FHTs is instead rigidly connected to the chassis. Geometric and inertial main characteristics (for condition 1) of tested FTHs are reported in Table 1. The

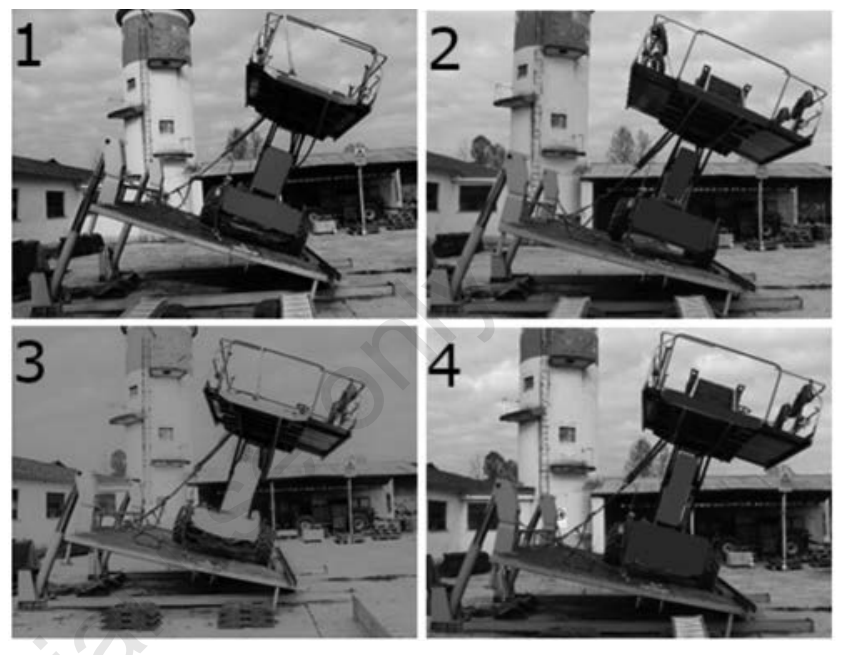

Figure 2. Rollover experimental campaign. Condition 1, 2, 3 and 4.

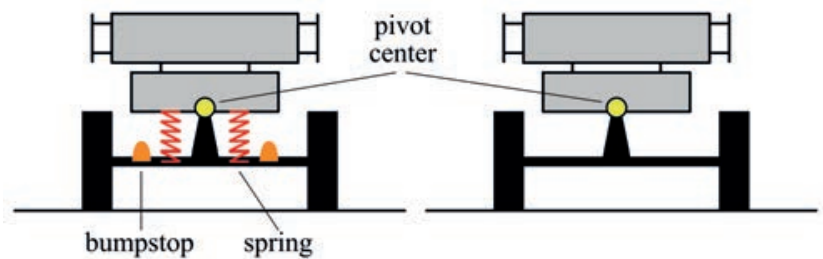

with swivel limiting device

without swivel limiting device

Figure 3. Front swivelling axle scheme, with and without limiting device.

Table 1. Main geometrical and mass data of the two tested fruit harvesting trucks (FHTs).

\begin{tabular}{lccc} 
Parameter & Unit & FHII & FHI2 \\
Mass & $\mathrm{kg}$ & 2260 & 2490 \\
c.o.g. height from ground & $\mathrm{mm}$ & 1256 & 1540 \\
\hline c.o.g. distance from rear axle & $\mathrm{mm}$ & 780 & 703 \\
Wheelbase & $\mathrm{mm}$ & 1948 & 1700 \\
\hline Front track width & $\mathrm{mm}$ & 1653 & 1670 \\
Rear track width & $\mathrm{mm}$ & 1653 & 1690 \\
\hline Minimum cargo bed height & $\mathrm{mm}$ & 1060 & 1000 \\
Maximum cargo bed height & $\mathrm{mm}$ & 2590 & 2920 \\
\hline Side platform max. opening & $\mathrm{mm}$ & 750 & 800 \\
Swivelling front axle & & Yes & Yes \\
\hline Swivelling limiting device & & Yes & No \\
\hline
\end{tabular}


position of the vehicle c.o.g. was obtained by measuring the force on each wheel by means of load scales while the vehicle was still on a flat ground and measuring the load on the rear wheel when the front of the vehicle was elevated thanks to an overhead crane as specified by standard ISO 789-6.

Making reference to condition 1, FHT2 has a higher mass (230 $\mathrm{kg}$ ) and a higher c.o.g. (284 mm). FHT2 presents also an higher lateral displacement of side platforms $(50 \mathrm{~mm})$ and an higher maximum height of the cargo bed $(330 \mathrm{~mm})$.

Results of the rollover tests performed on the two FHTs are reported in the Tilting tests section in direct comparison with the results of the developed $\mathrm{MB}$ model.

\section{Multibody model}

Collected experimental data for rollover of FHT allowed the development of MB model of the vehicle. The model was used to perform preliminary analysis of rollover as a function of several geometrical, inertial and stiffness parameters such as the height of the FHT cargo bed, the mass distribution due to workers and fruit bin, the tires stiffness, etc.

Analysis was carried out considering different tilting directions (which cannot be performed easily on the experimental tilting platform), i.e. during simulations the vehicle was tilted about an axis, which is non-parallel to the vehicle longitudinal axis.

\section{Model description}

The MB model, reported in Figure 4, was developed in Matlab Simulink environment with SimMechanics library. It consists in: i) the tilting platform that can be rotated about an axis, which can be turned about ground vertical axis. This allows to simulate combined roll and pitch rollover conditions; ii) the vehicle chassis; iii) the rear axle which is rigidly connected to the chassis; iv) the swivelling front axle which is connected to chassis through a pin; in case of FHT1 a nonlinear spring-damper element reproduces the visco-elastic behaviour of the suspension equipping the vehicle, including the effect of bumpstops. In other words, the stiffness increases as the axle gets closer to the vehicle chassis. In the case of FHT2, not equipped with a suspension system, the nonlinear spring-damper element only avoids interpenetration between front axle and chassis when relative rotation becomes large. No force is instead provided if relative rotation is small (less than $10^{\circ}$ ); v) the lifting cargo bed that is connected to the chassis by two bodies representing the bellow mechanism; vi) the bellow mechanism made of two rigid frames; vii) the opening side platforms that can slide along y direction with respect to cargo bed.

In order to reproduce the load of the truck other bodies are added to the model: i) the fruit bin, which is rigidly connected to cargo bed; ii) the workers who are rigidly connected to side platforms.

The tire is a key component of the system and has to be modelled properly (Keen et al., 2013; Melzi et al., 2014). To the purpose of the performed analysis, the tire is modelled as a visco-elastic component that can exchange vertical, longitudinal and lateral forces with the tilting table. It has also to allow the rotation of the hub along yaw pitch and roll axis with respect to the tilting table. The joint that provides the desired behaviour is a bushing joint in which the vertical forces $F_{z}$ are computed as follow:

$F_{z}=\left\{\begin{array}{r}k_{z} z+r_{z} \dot{z}, z<0 \\ 0, z \geq 0\end{array}\right.$ where $z$ is the vertical deflection of the tire:

$z=R_{0}-R$

with $R_{0}$ the unloaded tire radius and $R$ the effective tire radius while $k_{z}$ and $r_{z}$ are respectively the vertical stiffness and damping coefficient of the tire.

The vertical stiffness of the tire $k_{z}$ is of paramount importance in the rollover process since it allows the c.o.g. of the vehicle to move laterally while the vehicle is tilted. This effect increases the risk of rollover reducing the maximum allowed tilting angle at which the vehicle experiences rollover.

Longitudinal $F_{x}$ and lateral $F_{y}$ forces are modelled in order to be saturated according to static friction coefficient $\mu$ between the tire and the road (platform in case of simulations of the tilting tests). Modelling the tyre compliance by means of longitudinal and lateral springs connecting the wheel rim to the contact patch, the resulting contact force on the rim read:

$$
F_{x}=\left\{\begin{array}{r}
k_{x} x+r_{x} \dot{x},\left|k_{x} x+r_{x} \dot{x}\right|<\mu F_{z} \\
\mu F_{z},\left|k_{x} x+r_{x} \dot{x}\right| \geq \mu F_{z}
\end{array}\right.
$$

$F_{y}=\left\{\begin{array}{r}k_{y} y+r_{y} \dot{y},\left|k_{y} y+r_{y} \dot{y}\right|<\mu F_{z} \\ \mu F_{z},\left|k_{y} y+r_{y} \dot{y}\right| \geq \mu F_{z}\end{array}\right.$

with $x$ and $y$ the lateral displacement of tire contact point in longitudinal and lateral directions, $k_{x}$ and $k_{y}$ the longitudinal and lateral stiffness of the tire carcass and $r_{x}$ and $r_{y}$ the damping coefficients.

\section{Model validation}

The MB model of the FHT was validated according to available experimental data of FHT1 and FHT2. Figure 5 shows an example of rollover simulation performed with the developed MB model.

The first set of performed simulations aimed to reproduce the experimental setup in order to compare the rollover angle thus to

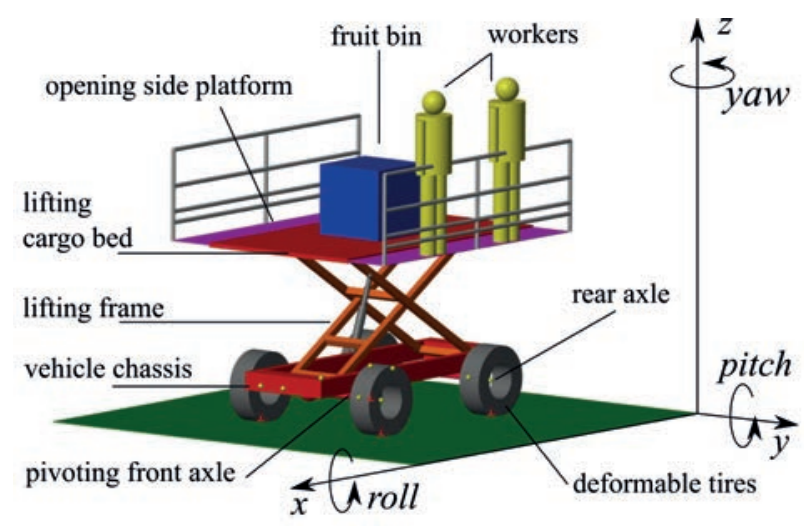

Figure 4. Multibody model of fruit harvesting truck for rollover simulation. 
validate the MB model. Table 2 reports the identified values of tyre and suspension stiffness.

Tables 3 and 4 report the comparison between experimental data and simulation results for the four considered load conditions for FHT1 and FHT2 respectively. It is to point out that: i) FHT1 has a swivelling axle with angle limiting device. The swivelling angle limiting device of FHT1 consists in two helical springs. This means that the rollover of the vehicle starts with the lifting of the upstream wheel of the non-swivelling axle but the full rollover is obtained when also the upstream wheel of the swivelling axle is detached from the ground; ii) for FHT2, instead, the rollover condition is reached when the first wheel (the upstream wheel of the non-swivelling axle) is detached from the ground and, since no swivelling device is present that can stop the rollover as it happens for truck like FHT1, the rolling inertia of the truck lead to complete rollover when the swivelling axle rotates bumping into bump stops. It can be noticed that there is a good agreement between simulation and experimental data both for FHT1 and FHT2. For FHT1 both the detachment of the $1^{\text {st }}$ (partial rollover) and $2^{\text {nd }}$ wheel (complete rollover) are reported.

The differences in simulation predicted rollover angle and experimental data is in general lover than $5 \%$. The biggest difference is in longitudinal rollover of FHT2 with an error of about $10 \%$. More in details, it can be noticed from Table 3 how the model is capable of correctly reproduce the rollover of FHT1 where the contact loss of $1^{\text {st }}$ and $2^{\text {nd }}$ wheel is correctly reproduced. From these tests emerges that FHTs without suspension are more critical by the point of view of rollover due to the absence of swivelling limiting device and due to an higher c.o.g.. $1^{\text {st }}$ wheel detachment of FHT1 is comparable with FHT2 rollover. As expected, the most critical condition for both FHTs is the one with asymmetrical load (condition 3), i.e. with two operators on the same side of the vehicle, while the presence of the bin at the centre of the cargo bed slightly affects the rollover attitude of the vehicle.

\section{Results of combined rollover simulations}

Once the MB model has been validated it is used to perform simulations of combined pitch and roll tilting and to study the influence of model parameters on rollover.

In order to study the rollover stability of the vehicle due to different orientation of the tilting angle, the vehicle is placed over the tilting platform with different value of angle $\Psi$ which is the angle between the tilting platform inclination axis and the vehicle forward axis as illustrated in Figure 5. This allows studying the effect of combined rollover due to a forward and lateral tilting.

The tilting axis rotates about the world $\mathrm{z}$-axis of a given angle $\Psi$ which is null when the tilting axis is aligned with vehicle forward axis ( $\mathrm{x}$ axis). This means that when $\Psi=0$ the vehicle is tilted laterally (parallel to roll axis) while when $\Psi=\pi / 2$ the vehicle is tilted forward so to simulate longitudinal rollover.

Figure $6 \mathrm{~A}$ and $\mathrm{B}$ respectively report the combined rollover simulation results of FHT1 for $1^{\text {st }}$ and $2^{\text {nd }}$ wheel detachment from ground. The graphs are in polar coordinates so that the angular coordinate corresponds to the tilting axis orientation $\Psi$ while the radial coordinate corresponds to the rollover angle in degrees.

Figure $6 \mathrm{C}$ reports same simulation results for FHT2 where only the complete rollover is reported.

From reported figures, the following considerations arise: i) for FHT1 the worst condition is in case of pure lateral rollover ( $\Psi$ $=0^{\circ}$ ); ii) for FHT2, instead, the worst condition occurs when the tilting axis is rotated of about 25 degrees $\left(\Psi=25^{\circ}\right)$; iii) lateral rollover $\left(\Psi=0 / 180^{\circ}\right)$ is more critical than longitudinal rollover $(\Psi$ $\left.= \pm 90^{\circ}\right)$ in all working conditions; iv) for both trucks, the longitudinal rollover is more critical if rear axle is downstream (tilting axis $\Psi=270^{\circ}$ ); v) as expected, FHT2 rolls over for smaller angle

Table 2. Stiffness values used in multibody model.

\begin{tabular}{lcccc} 
Description & Symbol & Unit & Value FHI1 & Value FHI2 \\
Tyre vertical stiffness & $K_{z}$ & $\mathrm{~N} / \mathrm{mm}$ & 400 & 375 \\
Tyre longitudinal stiffness & $K_{x}$ & $\mathrm{~N} / \mathrm{mm}$ & 300 & 375 \\
\hline Tyre lateral stiffness & $K_{y}$ & $\mathrm{~N} / \mathrm{mm}$ & 300 & 375 \\
Suspension roll stiffness & $K_{\phi}$ & $\mathrm{Nm} / \mathrm{deg}$ & 910 & 0 \\
\hline
\end{tabular}

Table 3. Rollover angle for fruit harvesting truck 1, comparison between experimental and simulation for different load conditions. 1 st and $2^{\text {nd }}$ refers to $1^{\text {st }}$ and $2^{\text {nd }}$ wheel detachment from ground.

\begin{tabular}{|c|c|c|c|}
\hline Condition & Rollover type & Exp. (deg) & Sim. (deg) \\
\hline 1 & $\begin{array}{r}\text { Lateral }\left(1^{\text {st }}\right) \\
\left(2^{\text {nd }}\right)\end{array}$ & $\begin{array}{l}20.3 \\
26.3\end{array}$ & $\begin{array}{l}21.0 \\
27.6\end{array}$ \\
\hline 2 & $\begin{array}{r}\text { Lateral }\left(1^{\text {st }}\right) \\
\left(2^{\text {nd }}\right)\end{array}$ & $\begin{array}{l}16.1 \\
21.7\end{array}$ & $\begin{array}{l}16.2 \\
20.2\end{array}$ \\
\hline 3 & $\begin{array}{r}\text { Lateral }\left(1^{\text {st }}\right) \\
\left(2^{\text {nd }}\right)\end{array}$ & $\begin{array}{l}12.2 \\
18.7\end{array}$ & $\begin{array}{l}12.4 \\
18.1\end{array}$ \\
\hline 43 & $\begin{array}{r}\text { Latera }\left(1^{\text {st }}\right) \\
\left(2^{\text {nd }}\right)\end{array}$ & $\begin{array}{l}13.9 \\
20.9\end{array}$ & $\begin{array}{l}13.8 \\
20.4\end{array}$ \\
\hline
\end{tabular}

Table 4. Rollover angle for fruit harvesting truck 2, comparison between experimental and simulation for different load conditions.

\begin{tabular}{lccc} 
Condition & Rollover type & Exp. (deg) & Sim. (deg) \\
1 & Longitudinal & 24.8 & 27.3 \\
& Lateral & 20.0 & 21.6 \\
2 & Lateral & 14.8 & 15.5 \\
\hline 3 & Lateral & 11.9 & 11.9 \\
4 & Lateral & 13.3 & 13.0 \\
\hline
\end{tabular}
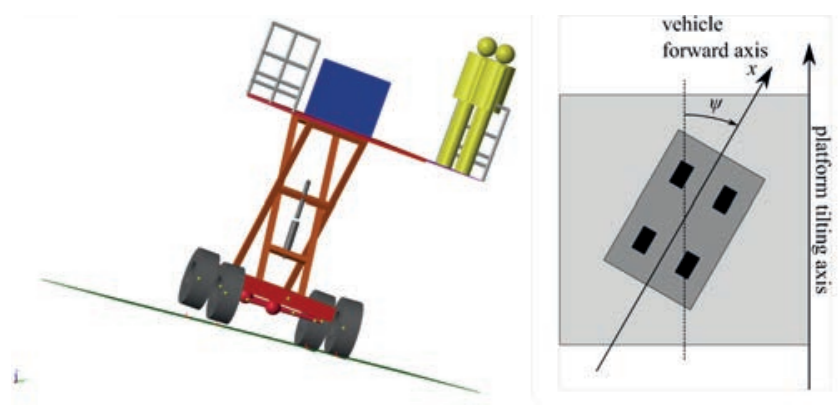

Figure 5. Multibody model rollover simulation example of working load condition 3 in combined tilting and representation of platform tilting axis. 
than FHT1, this is due to the absence of swivelling angle limiting device on the pivoting axle and due to a higher c.o.g.; vi) rollover angle of FHT2 is comparable with the tilt angle producing the detachment of the 1st wheel (rear upstream wheel) of FHT1; vii) therefore, the swivelling angle limiting device on the pivoting axle plays an important role in increasing the rollover stability of the vehicle and, obviously, the effect is important for prevalent lateral rollover; in pure longitudinal rollover the device does not affect the rollover condition; viii) working load conditions 1 and 2 present the same rollover risk for right and left lateral rollover, this is due to the symmetry of the mass distribution; on the other hand, working load conditions 3 and 4 show an asymmetrical behaviour due to the unbalance of the load ( 2 workers on the same side of the truck and none on the other); ix) for both the trucks, working load condition 3 is the worst one in case of lateral rollover when the loaded side is downstream and $\Psi$ is in the range $-0^{\circ}: 60^{\circ}$; $x$ ) conversely, when the loaded side is upstream the worst working load condition is number 2 for both trucks.

\section{Model parameters influence on rollover}

Standard ISO 16231-2 states, in fact, that the most critical condition for rollover occurs for a combination of pitch and roll rotations of the vehicle, depending on the geometry of the front axle suspension. Table 5 reports the most critical tilting angle inclination $\Psi$ computed according to standard and obtained by simulations. In particular, the most critical rollover condition, for vehicle without swivelling limiting device on the pivoting axle (as it is for
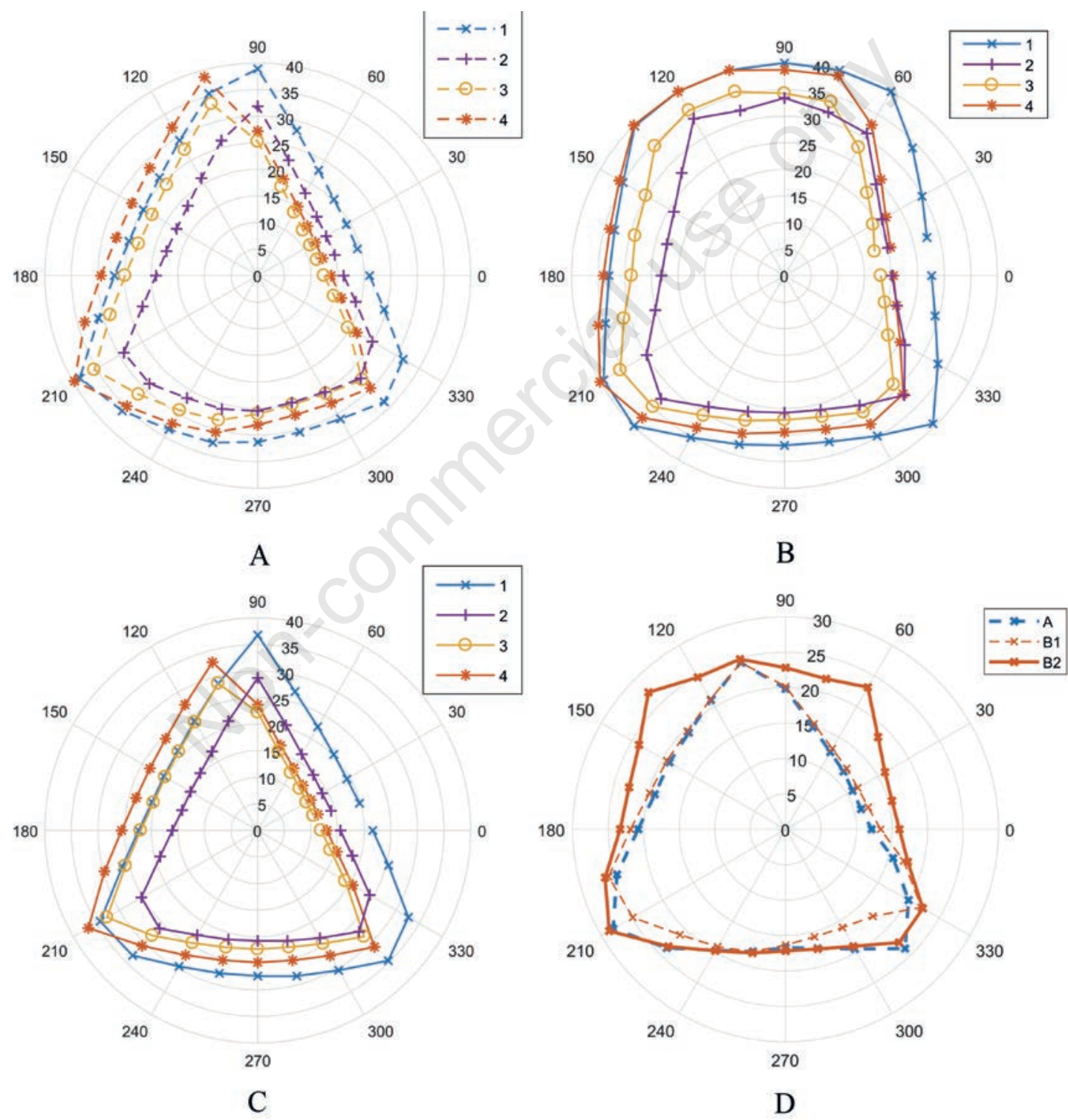

Figure 6. Rollover of FHT1, $1^{\text {st }}(\mathrm{A})$ and $2^{\text {nd }}(\mathrm{B})$ wheel detachment from ground. The blue -x-curves refers to load condition 1 , the magenta -+- curve refers to load condition 2 , the yellow -o- curve refers to load condition 3 and finally red curve refers to load condition 4. C) Rollover of FHT2. The blue -x- curves refers to load condition 1, the magenta -+- curve refers to load condition 2, the yellow o- curve refers to load condition 3 and finally red curve refers to load condition 4. D) Rollover angle of FHT2 without suspension springs (A) and with springs on the front suspension: $1^{\text {st }}$ and $2^{\text {nd }}$ detachment (B1 and B2 respectively). 
FHT2), is obtained for a tilting angle, which is parallel to the edge of the triangle obtained projecting on the ground the axis passing from vehicle c.o.g. and pivoting point of the swivelling axle (see ISO 16231-2 for further details).

Since FHTs without suspensions resulted to be the most critical, further simulations were performed in order to deeper investigate the effect of front axle suspension and tire stiffness.

\section{Suspension system influence on rollover}

Suspension stiffness of the pivoting axle revealed to play an important role in evaluation of rollover. To better evaluate the effect of suspension springs, rollover simulations were performed. FHT2 MB model was used with and without front axle suspension. Only the most critical working load condition (3) was simulated. Figure 6D shows the results of rollover simulation for this condition. Reported lines are representative of the rollover without suspension springs (line A), and the $1^{\text {st }}$ and $2^{\text {nd }}$ wheel detachment with suspension springs (lines B1 and B2 respectively). From simulation results emerges that: i) the $1^{\text {st }}$ detachment is slightly affected by front axle suspension. In particular, the most relevant differences are in ranges $195^{\circ}<\Psi<235^{\circ}$ and $270^{\circ}<\Psi<330^{\circ}$. In case of prevailing lateral rollover, instead, the difference between the two cases is marginal; ii) considering the complete rollover, the presence of the suspension springs highly increases the rollover angle in particular in ranges $-15^{\circ}<\Psi<90^{\circ}$ and $120^{\circ}<\Psi<180^{\circ}$.

Table 5. Fruit harvesting truck 2 rollover angle $\rho$ and tilt axis inclination $\Psi$. Comparison between simulation and standard calculated values.

\begin{tabular}{lcccc}
\hline Working load condition & $\rho$ sim & $\rho$ std & $\Psi$ sim & $\Psi$ std \\
\hline 1 & 18.26 & 19.81 & 22.5 & 22.3 \\
\hline 2 & 13.92 & 14.74 & 23.5 & 24.0 \\
3 & 11.68 & 11.96 & 25.0 & 25.0 \\
\hline 4 & 10.83 & 11.40 & 25.0 & 24.8 \\
\hline
\end{tabular}

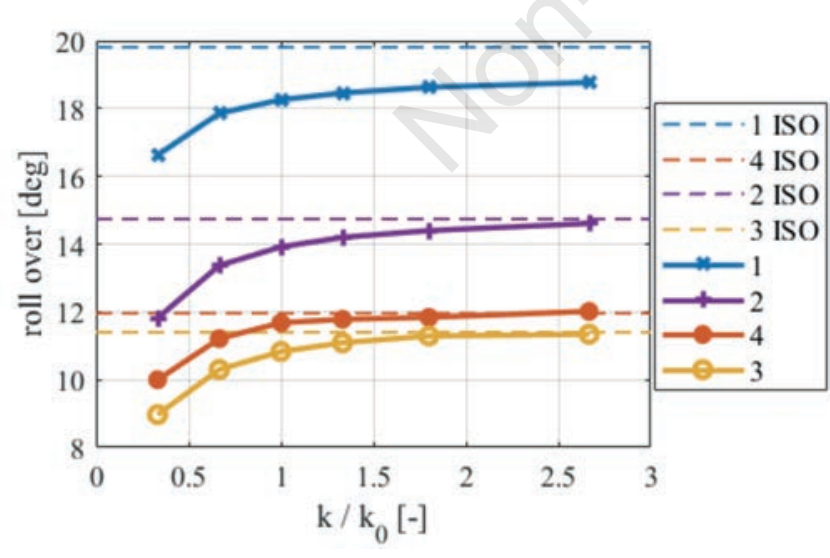

Figure 7. Rollover angle of FHT2 for different value of tire stiffness (on the $\mathrm{x}$ axis the values have been normalised with respect to experimentally identified tire stiffness). 1,2,3 and 4 represent the working conditions simulated through multibody model while 1,2,3 and 4 ISO represent the calculated valued according to ISO prescription.

\section{Tire-soil stiffness influence on rollover}

Another effect, which resulted interesting to investigate, was the effect of tire-soil stiffness influence on rollover. The standard does not consider it while it may play an important role in rollover.

Table 5 reports the values of minimum rollover angle $(\rho)$ and the tilting platform rotation $(\Psi)$ at which rollover occurs during simulation and according to ISO standard.

As it can be seen, there is a general agreement between MB simulations and results predicted by ISO standard.

To understand the influence of tire stiffness on rollover, several simulations were performed changing the value of the tire stiffness $k_{z}$. It is to point out that also longitudinal and lateral stiffness were changed accordingly in order to reproduce the effect of inflating pressure of the tire on its stiffness along all the directions.

Figure 7 reports the rollover angle for most critical tilting axis ( $\Psi$ ) for all working load conditions as a function of normalised tire stiffness. Tire stiffness is made non-dimensional by dividing it by the nominal value $\left(k_{0}\right)$ used during simulations shown in the Results section. It can be noticed that, increasing the value of tire stiffness, the rollover angle reaches asymptotically the rollover value calculated according to standard ISO 16231-2.

The rollover angle, for identified stiffness value $k_{0}$, is between 3 and $8 \%$ lower than the value obtained by standard calculation. This difference increases up to $10 \%$ if the stiffness of the tire is reduced of about $30 \%$.

\section{Conclusions}

In the present paper, an investigation concerned with rollover of FHT was presented, based on an experimental-numerical approach.

Experimental field tests allowed the identification of typical operating conditions for these vehicles.

Experimental tests using a tilting table instead allowed evaluating FHT rollover limit in the most interesting working conditions identified during field tests. Based on the results of tilt-table test, a MB of the FHT was developed and validated. The MB model reproduces the main characteristics of a FHT: the moving cargo bed, the telescopic platforms, and the variable position of operators, buckets and load. Moreover, the MB model accounts for compliance of tires, suspension of the front axle (if any) and joints.

The developed MB model of the FHT was then used to analyse the influence of vehicle parameters on rollover limit about a generic tilt axis (i.e. not purely lateral or longitudinal). Specifically, the influence of position of loads on the cargo bed, front axle suspension and tire compliance was investigated. Simulation results stated that, as expected, the most critical load condition is the one that foresees the presence of the full bin at the cargo bed centre with the operators standing on the downstream side (no one at the upstream one). For FHTs equipped with a front axle suspension, lateral rollover is always the most critical condition (tilting axle perpendicular to the vehicle longitudinal axis); on the contrary, lateral rollover is not the most critical conditions for FHTs not equipped with any front axle suspension. The rotation of the tilt axis at which the most critical rollover condition occurs depends on vehicle geometry and mass distribution.

Finally, compliance of tires may significantly reduce rollover limit (as calculated according to standard ISO). 


\section{References}

Cavallo E., Görücü S., Murphy D.J. 2015. Perception of side rollover hazards in a Pennsylvania rural population while operating an all-terrain vehicle (ATV). Work 51:281-8.

Coppock G.E., Jutras P.J. 1960. An investigation of the mobile picker's platform approach to partial mechanisation of citrus fruit picking. Florida State Horticult. Soc. 258-53.

Fathallah A.F. 2010. Musculoskeletal disorders in labor-intensive agriculture. Appl. Ergon. 41:738-43.

Franceschetti B., Lenain R., Rondelli V. 2014. Comparison between a rollover tractor dynamic model and actual lateral tests. Biosyst. Engine. 127:79-91.

Görücü S., Cavallo E., Murphy D.J. 2014. Perceptions of tilt angles of an agricultural tractor, J. Agromed. 19:5-14.

Gravalos I., Gialamas T., Loutridis S., Moshou D., Kateris D., Xyradakis P., Tsiropoulos Z. 2011. An experimental study on the impact of the rear track width on the stability of agricultural tractors using a test bench. J. Terramechan. 48:319-23.

Jung D., Jeong J., Woo S.M., Jang E., Park K., Son J. 2013. A study on the stability of a vehicle with lifting utility. Adv. Mater. Res. 753. Trans Tech Publications.

Keen A., Hall N., Soni P., Gholkar M.D., Cooper S., Ferdous J. 2013. A review of the tractive performance of wheeled tractors and soil management in lowland intensive rice production. J. Terramechan. 50:45-62.

Liu J., Ayers P.D. 1999. Off-road vehicle rollover and field testing of stability index. J. Agricult. Safety Health. 5:59-71.

Melzi S., Negrini S., Sabbioni E. 2014. Numerical analysis of the effect of tire characteristics, soil response and suspensions tun- ing on the comfort of an agricultural vehicle. J. Terramechan. 55:17-27.

Vidoni R., Bietresato M., Gasparetto A., Mazzetto F. 2015. Evaluation and stability comparison of different vehicle configurations for robotic agricultural operations on side-slopes. Biosyst. Engine. 129:197-211.

Wang W., Wu T., Hohimer C., Mo C., Zhang Q. 2016. Stability analysis for orchard wearable robotic system. IFACPapersOnLine 49:61-5.

ISO. 1982. Agricultural tractors - Test procedures - Part 6: Centre of gravity. ISO 789-6:1982. International Organisation for Standardisation Publ., Geneva, Switzerland.

ISO. 2007. Earth-moving machinery - Physical dimensions of operators and minimum operator space envelope. ISO 3411:2007. International Organisation for Standardisation Publ., Geneva, Switzerland.

ISO. 2013. Self-propelled agricultural machinery - Assessment of stability — Part 1: Principles. ISO 16321-1:2013. International Organisation for Standardisation Publ., Geneva, Switzerland.

ISO/FDIS. 2015. Self-propelled agricultural machinery Assessment of stability - Part 2: Determination of static stability and test procedures. ISO 16321-2:2015. International Organisation for Standardisation Publ., Geneva, Switzerland.

UNI EN. 2010. Safety of industrial trucks - Self propelled variable reach trucks. UNI EN 1459:2010.

UNI ISO. 2008. Industrial trucks — Verification of stability Part 1: General - Part 2: Counterbalanced trucks with mast. ISO 22915-1/2:2016. International Organisation for Standardisation Publ., Geneva, Switzerland. 